\title{
Microenvironment Dependent Photobiomodulation on Function-Specific Signal Transduction Pathways
}

\author{
Timon Cheng-Yi Liu, ${ }^{1}$ De-Feng Wu, ${ }^{1}$ Ling Zhu, ${ }^{1}$ P. Peng, ${ }^{1}$ Long Liu, ${ }^{2}$ and Xiang-Bo Yang ${ }^{1,3}$ \\ ${ }^{1}$ Laboratory of Laser Sports Medicine, South China Normal University, University Town, Guangzhou 510006, China \\ ${ }^{2}$ Department of Chemistry and Biology, College of Science, National University of Defense Technology, Changsha 410073, China \\ ${ }^{3}$ MOE Key Laboratory of Laser Life Science and Institute of Laser Life Science, College of Biophotonics, South China Normal University, \\ Guangzhou 510631, China
}

Correspondence should be addressed to Timon Cheng-Yi Liu; liutcy@scnu.edu.cn

Received 7 January 2014; Accepted 28 January 2014; Published 9 March 2014

Academic Editor: Quan-Guang Zhang

Copyright (C) 2014 Timon Cheng-Yi Liu et al. This is an open access article distributed under the Creative Commons Attribution License, which permits unrestricted use, distribution, and reproduction in any medium, provided the original work is properly cited.

Cellular photobiomodulation on a cellular function has been shown to be homeostatic. Its function-specific pathway mechanism would be further discussed in this paper. The signal transduction pathways maintaining a normal function in its functionspecific homeostasis (FSH), resisting the activation of many other irrelative signal transduction pathways, are so sparse that it can be supposed that there may be normal function-specific signal transduction pathways (NSPs). A low level laser irradiation or monochromatic light may promote the activation of partially activated NSP and/or its redundant NSP so that it may induce the second-order phase transition of a function from its dysfunctional one far from its FSH to its normal one in a function-specific microenvironment and may also induce the first-order functional phase transition of the normal function from low level to high level.

\section{Introduction}

Cellular photobiomodulation (PBM) is a modulation of laser irradiation or monochromatic light (LI) on cells in vitro or in vivo or ex vivo, which stimulates or inhibits cellular functions but does not result in irreducible damage. The LI intensity is in the range of about $10-1000 \mathrm{~mW} / \mathrm{cm}^{2}[1,2]$. As we have classified [3-5], the LI used in PBM is always a low intensity LI (LIL), $\sim 10 \mathrm{~mW} / \mathrm{cm}^{2}$, which includes the LI used in the so-called ultra-low-level laser therapy [6], but moderate intensity LI (MIL), $0.10 \sim 1.0 \mathrm{~W} / \mathrm{cm}^{2}$, is of PBM if the irradiation time is not so long that it irreducibly damages organelles or cells. The PBM of LIL and MIL are denoted as LPBM and MPBM, respectively. The MIL with short irradiation time and LIL are two kinds of well-known low level LI (LLL).

Many studies have focused on signal transduction pathway mediated cell-autonomous mechanisms of PBM. By contrast, we proposed that the cellular microenvironment confers the response of cellular signal transduction pathways to LLL. Many cellular signal transduction pathways may be modulated by LLL, but which signal transduction pathway can be modulated has been left to be resolved. Many studies suggested that the modulated signal transduction pathway may depend on the microenvironment in which cells live. It would be reviewed in this paper.

\section{Function-Specific Homeostasis}

Negative feedback is common in biological processes and acts to optimize the activity of a circuit in the presence of alleles with altered activities [7], which can maintain a system's stability to internal and external perturbations. Function-specific homeostasis (FSH) is a negative-feedback response of a biosystem to maintain the function-specific conditions inside the biosystem so that the function is perfectly performed $[3,4,8,9]$. A function in/far from its FSH is called a normal/dysfunctional function. A normal function is better performed than all the dysfunctional functions so that the normal function is locally the best performed function. 
The phenomena are the well-known "Arndt-Schulz Law" or the J-shaped curves. Moreover, the normal function can resist external perturbations so that the peak of Arndt-Schulz Law or the J-shaped curve can be extended to a plateau so that it is called Arndt-Schulz Plateau Law or the U-shaped curve. In $10 \%$ fetal bovine serum (FBS), it has been found that there are the normal glucose $(\mathrm{nG})$ at about $22.5 \mathrm{mM}$, in which many cell lines such as C2C12 [10, 11] and C3H10 T1/2 [12] proliferate at its optimal rate, and the low/high glucose $(\mathrm{G} / \mathrm{hG})$ whose concentration was lower/higher than the one of $\mathrm{nG}$ and in which the cell lines proliferate at a rate lower than the optimal rate. In other words, the cells in $\mathrm{nG}$ and $10 \%$ FBS are in their respective proliferation-specific homeostasis (PlSH).

A normal function can resist external/internal disturbance under its threshold. Straussman et al. [13] have studied the effects of twenty-two cytokines, each at five concentrations, on six melanoma cell lines in their respective $\mathrm{PlSH}$, respectively. They found that only hepatocyte growth factor (HGF) upgraded the normal proliferation but all the other twenty-one cytokines cannot affect the normal proliferation. That is suggested that only HGF was allowed to activate its signal transduction pathway, but all the other twentyone cytokines were not allowed to activate their pathways, respectively.

The experimental reproducibility in a PISH is intrinsic. In a recent analysis, Haibe-Kains et al. [14] reported that the measured drug response data are highly discordant for the two large-scale pharmacogenomic studies, the Cancer Genome Project (CGP), and the Cancer Cell Line Encyclopedia (CCLE). CGP and CCLE cells were grown in Roswell Park Memorial Institute (RPMI) or Dulbecco's Modified Eagle's Medium (DMEM)/F12 medium with 5\% and 10\% FBS, respectively. This suggested that each CGP cell line was far from its respective PlSH and its $\mathrm{IC}_{50}$ (concentration in micromolar at which the drug inhibited $50 \%$ of the maximum cellular growth) against one antidrug cannot be reproducible. As reported by the CGP, there was only a fair correlation between camptothecin $\mathrm{IC}_{50}$ measurements generated at two sites using matched cell line collections and identical experimental protocols. On the other hand, each CCLE cell line may be in its respective $\mathrm{PISH}$ and its $\mathrm{IC}_{50}$ value against one antidrug can be reproducible. This was why there were more than one $\mathrm{IC}_{50}$ value of each CGP cell line against one antidrug for each $\mathrm{CCLE}_{\mathrm{IC}_{50}}$ value and the vast majority of drugs and gene-drug associations then yielded poor concordance for $\mathrm{IC}_{50}$ and AUC (area under the activity curve measuring dose response).

\section{Microenvironments}

Cells may have many functions. Which function may be perfectly performed depends on the microenvironment in which cells live. Cells in a serum-free medium may become apoptotic and can be modulated by a LLL [15], but they cannot proliferate, attach, or migrate, so that the PBM on their proliferation $[16,17]$, attachment $[16]$, or migration [16] could not be observed. Many studies have focused on cellautonomous mechanisms of drug resistance, but Straussman et al. [13] found that the tumor microenvironment conferred innate resistance to therapy. They found that stromal cell secretion of HGF resulted in activation of the HGF receptor MET, reactivation of the mitogen-activated protein kinase (MAPK) and phosphatidylinositol-3-OH kinase- (PI3K-) AKT signaling pathways, and immediate resistance to RAF inhibition.

A cell may only perform the function in a functionspecific microenvironment. Eduardo et al. [18] have studied human dental pulp stem cell (DPSC) proliferation in 5\%, 10\%, $12.5 \%$, and $15 \%$ FBS, respectively, and found that there was no significant difference of the proliferation in $10 \%$ FBS from the one in $12.5 \%$ FBS after $24 \mathrm{~h}$, both of which were significantly smaller than the one in $15 \%$ FBS, but larger than the one in $5 \%$ FBS. It indicated that the proliferation in $10 \%$ or $12.5 \%$ FBS was in its $\mathrm{PISH}$, resisting FBS change. For many cell lines in $10 \%$ FBS, only proliferation can be performed and the medium is called a proliferation-specific microenvironment (PSM). For many cell lines, proliferation in 10\% FBS and $\mathrm{nG}$ is in its $\mathrm{PlSH}$ in $\mathrm{nG}(\mathrm{nPlSH})$, and the microenvironment is called a $\mathrm{PISH}$-specific microenvironment (PISM). If the $\mathrm{C} 3 \mathrm{H} 10 \mathrm{~T} 1 / 2$ cells were cultivated in $10 \% \mathrm{FBS}$ and $\mathrm{lG}$ such as 0 or $5 \mathrm{mmol} / \mathrm{L}$ glucose or hG such as 100,200 , or $300 \mathrm{mmol} / \mathrm{L}$ glucose for 3 or $6 \mathrm{~h}$ and then cultivated in a PISM for 8 days, the modulated proliferation would become the normal proliferation [12].

A cell may become apoptotic in a PSM or a differentiation-specific microenvironment (DSM). A LLL can inhibit apoptosis by promoting proliferation in a PSM [19] or promoting differentiation in a DSM [20]. Many groups have studied the proliferation-mediated apoptosis inhibition, but only Zhu et al. [20] have studied the differentiation-mediated apoptosis inhibition. Moreover, the latter inhibition was more effective than the former inhibition. As a fact, the in vivo neurons are mostly differentiated. The differentiationmediated apoptosis inhibition of PBM should be widely studied.

\section{Function-Specific Pathways}

Signal transduction pathways are always signal-dependent. However, it may not work when the cells are in their FSH [13]. Cancer cells in their respective PISH eliminate various signaling pathways, especially apoptotic, permitting their surviving, spreading, and thriving in "foreign" organs [21]. It suggested that the negative feedback of the cellular FSH not only resists internal/external disturbance but also resists the activation of many other irrelative signal transduction pathways. The signal transduction pathways are always found by destroying the FSH with serum deprivation or starvation or other stresses. For a cell far from its FSH, many signal transduction pathways have been partially activated [22]. One can alternatively find one of them so that one signal may have many alternative signal transduction pathways. The signal transduction pathways were studied from the viewpoint of functions in this section. 
4.1. Normal Function-Specific Signal Transduction Pathways. The activation of one signal transduction pathway can not only be directly promoted, but also be indirectly promoted by inhibiting the activation of other pathways. For example, insulin-like growth factor- (IGF-) 1 can restore dexamethasone- (DEX-) induced heart growth arrest in rats [23], and DEX can promote the IGF-1 promotion on skeletal muscle cell proliferation [24] and the IGF binding protein (IGFBP) 1 production of rat hepatocytes [25] in serum-free medium. The signal transduction pathway activation of a cell may also be inhibited by its FSH. The negative feedback of the cellular FSH can resist the activation of many other irrelative signal transduction pathways so that some signal transduction pathways may be left to be fully activated. It has been found that the best pathways for a dysfunctional function to become normal or a normal function to become upgraded are sparse [9]. Straussman et al. [13] found that the normal proliferation was maintained by full activation of the platelet-derived growth factor (PDGF) pathway, BRAF, MAPK kinase (MEK), and extracellular signal-regulated kinase (ERK), which not only resisted the proliferation effects of twenty-one cytokines, each at five concentrations, but also resisted their signal transduction pathway activation. At this point, it is reasonable to suppose that there may be normal function-specific signal transduction pathways (NSPs) so that the FSH can maintain the full activation of the NSPs but resist the activation of other irrelative signal transduction pathways. The PDGF pathway is the NSP of the normal proliferation of the six melanoma cell lines according to [13].

4.2. Redundant Signal Transduction Pathways. One normal function may have $N$ possible NSPs. Genetic redundancy means that $N$ genes are performing the same function and that inactivation of one of these $N$ genes has little or no effect on the biological phenotype [26]. The $N$ genes performing the same function are called redundant genes with one another [8]. Each redundant gene may have its NSP in its NSP-specific microenvironment (NSM). The full activation of each NSP can maintain its normal function in its NSM. The N NSPs of the same normal function are called redundant NSPs (rNSPs) with one another. Straussman et al. [13] found that HGF upgraded its normal proliferation of the six melanoma cell lines and further found that the proliferation enhancements were mediated by full activation of HGF pathway, PI3K, AKT or RAFl, MEK, and ERK and then by the synergistic full activation of both PDGF pathway and HGF pathway. The HGF pathway and the PDGF pathway are the two ones among the N NSPs of the normal proliferation of the six melanoma cell lines.

Whether NSP of a normal function actually maintains the normal function depends on the microenvironment. For a dysfunctional function, many signal transduction pathways have been partially activated [22]. While a dysfunctional function becomes a normal function, only sparse signal transduction pathways are left to be fully activated [9], but other irrelative signal transduction pathways are completely inhibited. The sparse signal transduction pathways are always one NSP. Liu et al. $[9,10]$ have studied the effects of hG at
$90 \mathrm{mM}$ and the LIL at $640 \mathrm{~nm}$ on the messenger ribonucleic acid (mRNA) of 6 genes of $\mathrm{C} 2 \mathrm{C} 12$ cells in their nPlSH and found that $h G$ decreased the proliferation and the mRNA expression of sirtuin 1 and manganese superoxide dismutase (MnSOD) but increased the mRNA expression of IGF-1, forkhead box O family (FOXO) 3a, Bcl-2 interacting mediator of cell death (Bim), and p27 and that LIL further increased IGF-1 mRNA expression but decreased Bim mRNA expression until it was lower than the one of $\mathrm{C} 2 \mathrm{C} 12$ cells in $\mathrm{nG}$ and completely recovered the proliferation and the mRNA expression of the other 4 genes so that the PISH in hG (hPlSH) was established. IGF-1 inhibits the mRNA of Bim [27, 28]. Obviously, IGF-1 pathway is the hG activated NSP (hNSP) of C2C12 myoblasts although the nG activated NSP (nNSP) has not been found yet. Both nPISH and hPISH maintain the same normal proliferation of $\mathrm{C} 2 \mathrm{C} 12$ myoblasts, but nNSP/hNSP maintains $\mathrm{nPISH} / \mathrm{hPlSH}$ in $10 \% \mathrm{FBS}$ and $\mathrm{nG} / \mathrm{hG}$.

4.3. Functional Phase Transitions. The normal DPSC proliferation in $10 \%$ FBS is maintained by its NSP. Eduardo et al. [18] found that MIL could not affect the normal DPSC proliferation in 15\% FBS but upgraded the one in 10\% FBS, and the upgraded one in $10 \%$ FBS was still significantly smaller than the one in 15\% FBS. This suggested that the one in $15 \%$ FBS may at least be maintained by the synergistic integration of NSP and one of its rNSPs. Generally, the full activation of each NSP maintains the first-order normal function in its NSM, and the synergistic full activation of one NSP and its $n-1$ rNSPs maintains the $n$ th-order normal function $(n=2,3, \ldots, N)$ in its NSM. The phase transition from a dysfunctional function to the first-order normal function is just the second-order functional phase transition, but the one from the $(n-1)$ th-order normal function to the $n$ th-order normal function is just the first-order functional phase transition. In our experiments [11], serum-shocked $\mathrm{C} 2 \mathrm{C} 12$ myoblasts were cultivated in $\mathrm{nG}$ and FBS at different concentrations. As FBS concentration increased, the dysfunctional proliferation becomes normal, and then the order of the normal proliferation becomes higher. Here, FBS induced a proliferation phase transition from the second-order one to the first-order one. In the experiments of Straussman et al. [13], PDGF pathway maintained the first-order normal proliferation in its PDGF pathway-specific medium, and the synergistic full activation of both PDGF pathway and HGF pathway maintained the second-order normal proliferation in its PDGF pathway-specific medium. Here, HGF induced a first-order proliferation phase transition of six melanoma cell lines in its PDGF pathway-specific medium.

4.4. Pathway-Mediated Photobiomodulation. There were many theories on precise molecular mechanism of PBM. Among them, the cytochrome $\mathrm{c}$ oxidase (COX) theory was very popular $[2,29]$. Cytochrome $c$ and COX represent the terminal step of the electron transport chain, the proposed rate-limiting reaction in mammals. Cytochrome $\mathrm{c}$ and COX show unique regulatory features including allosteric regulation, isoform expression, and regulation through cell signaling pathways [30]. The COX theory of PBM was 
proposed by Karu and Afanas'eva [31, 32]. It suggested that COX in mitochondria was the primary photoacceptor upon LLL exposure of cells, and PBM was mediated by LLL increased COX activity. According to the COX theory, LLL can increase nitric oxide (NO) production [2]. However, many studies [33-35] found that LIL may decrease the NO production. For example, Montoro et al. [35] found that LIL may decrease the NO production of FBS-deprived human dental pulp cells (HDPCs) without lipopolysaccharide (LPS) and FBS-deprived HDPCs with LPS. Moreover, $\mathrm{Wu}$ et al. [36] demonstrated that the initial reaction after photon absorption of MIL was photosensitization of COX, to inhibit enzymatic activity of COX in situ and cause respiratory chain reactive oxygen species (ROS) burst. Horvát-Karajz et al. [37] found that the effects of one time MIL irradiation, MIL induced stresses, were successful or self-limited at low dose but chronic at high dose according to our homeostasis theory $[3,4,8]$, and cytostatic drugs such as cytarabine, paclitaxel, and vincristine may change the successful stress of MIL at low dose into chronic stress. In this context, LLL cannot increase COX activity if COX was the primary photoacceptor.

As Wu et al. [36] have demonstrated, MPBM is indeed mediated by COX-mediated ROS $[4,38]$. According to our homeostasis theory $[3,4,8]$, we have suggested that the membrane receptors of cells or organelles were the primary photoreceptors of LIL, and LPBM was mediated by receptoractivated signal transduction pathways [4, 9, 38]. Several signaling pathways have been identified that target COX including protein kinase $\mathrm{A}$ and $\mathrm{C}$, receptor tyrosine kinase, and inflammatory signaling [30]. In addition, four phosphorylation sites have been mapped on cytochrome $c$ with potentially large implications due to its multiple functions including apoptosis, a pathway that is overactive in stressed cells but inactive in cancer. In other words, LIL-activated pathways may modulate COX activity so that it can explain LIL increased COX activity.

The LLL promotion of the normalization of a dysfunctional function may be mediated by the promotion of the activation of partially activated NSP or/and its rNSP. Miyata et al. [17] found that MIL promoted the phosphorylation of ERK $1 / 2$ between 5 and $30 \mathrm{~min}$ after MIL irradiation but did not affect p38 MAPK or c-Jun N-terminal kinase (JNK) phosphorylation. In our experiments $[9,10]$, the C2C12 myoblast proliferation in its nPISM is normal, but the one in $10 \% \mathrm{FBS}$ and $\mathrm{hG}$ at $90 \mathrm{mmol} / \mathrm{L}$ is dysfunctional. We found that hG increased IGF-1 mRNA expression of C2C12 myoblasts; LIL further increased the IGF-1 mRNA expression until the IGF-1 pathway is fully activated so that the hPISH was established.

\section{Photobiomodulation}

There are many cellular functions. Generally, a cellular microenvironment only allows one function to be performed. If the allowed function is dysfunctional, LPBM may promote the activation of its partially activated NSP until it is fully activated so that the dysfunctional function becomes normal.
If the allowed function is normal, for example, it is the $(n-1)$ th-order normal function $(n=2,3, \ldots, N)$ if the normal function has $N$ possible NSPs, which is maintained by the synergistic full activation of one NSP and its $n-2$ rNSPs in its NSM; LPBM may promote the activation of its partially activated $(n-1)$ th rNSP until it is fully activated so that the $(n-1)$ th-order normal function is upgraded to be the $n$ th-order normal function. The former and latter PBM are called direct and indirect PBM ( $\mathrm{PPBM}$ and iPBM), respectively. The $\mathrm{APBM}$ induces the second-order functional phase transition from a dysfunctional function to the firstorder normal function, but the iPBM induces a first-order functional phase transition from the $(n-1)$ th-order normal function to the $n$ th-order normal function $(n=2,3, \ldots, N)$ if the normal function has $N$ possible NSPs.

5.1. Direct Photobiomodulation. There have been many studies of dPBM on proliferation of cells, but few ones on the other cellular functions. There may be differentiationspecific homeostasis (DiSH). Zhu et al. [20] have found that the differentiation-mediated apoptosis inhibition of $\mathrm{dPBM}$ was mediated by brain-derived neurotrophic factor (BDNF). Saygun et al. [39] have found that the dPBM promotion of the osteoblast differentiation of human mesenchymal stem cells (MSCs) was mediated by basic fibroblast growth factor (bFGF).

Amyloid $\beta(\mathrm{A} \beta)[19,40]$ or 6-hydroxy dopamine [41] may decrease the proliferation rate by inducing apoptosis, and LIL $[19,40]$ or the insect antibacterial peptide [41], CopA3 (a Dtype disulfide dimer peptide, LLCIALRKK), may inhibit the apoptosis by promoting proliferation. In Meng et al.s paper [40], the neuron proliferation resisted LIL. It indicated that the neuron proliferation may be a normal proliferation. For the human neuroblastoma cell line SH-SY5Y in its PISH, $\mathrm{A} \beta$ decreased the proliferation rate, but LIL promoted the dysfunctional proliferation until it became normal [40] so that the $\mathrm{PISH}$ in $\mathrm{A} \beta(\mathrm{aPISH})$ was established. BDNF can stimulate neuronal proliferation [42]. Meng et al. [40] found that LIL increased BDNF level. LIL increased BDNF level of neurons and then promoted dysfunctional proliferation until the aPISH was established.

Huang et al. [43] exposed primary cultured murine cortical neurons to oxidative stressors: hydrogen peroxide, cobalt chloride, and rotenone in the presence or the absence of LIL at $810 \mathrm{~nm}$. They found that the LIL increased both MMP and ROS in nonoxidative neurons and increased MMP but reduced high ROS levels and protected cultured cortical neurons from death in oxidative cells. Huang et al. [34] further studied the effects of LIL at $810 \mathrm{~nm}$ on glutamate, N-methyl-D-aspartate (NMDA), or kainate induced excitotoxicity of primary murine cultured cortical neurons. They found that the measurements can be divided into two groups: those in which the effect of the LIL is similar in direction (both increased) regardless of whether the neurons are nonexcitotoxic or excitotoxic (these are viability, adenosine triphosphate (ATP), and mitochondrial membrane potential (MMP)) and those measurements in which the direction of the LIL effect is opposite, raised for nonexcitotoxic neurons 
and decreased for excitoxic neurons (these are intracellular $\mathrm{Ca}^{2+}, \mathrm{ROS}$, and NO). Their explanation based on the COX theory was very complicated $[34,43]$, but the one based on NSPs is very simple. Either oxidative/excitoxic or nonoxidative/nonexcitoxic neurons are dysfunctional so that their viability, ATP, and MMP have been promoted by LIL, but their mediated NSPs may be different from each other so that their intracellular $\mathrm{Ca}^{2+}$, ROS, and NO have been oppositely modulated by LIL although the NSPs may be redundant with each other.

Esmaeelinejad and Bayat [44] have studied LPBM on human skin fibroblasts in $1 G$ and 10\% FBS and found that LIL promoted the activation of interleukin-6 (IL-6)/bFGF mediated pathway. Jee et al. [45] found that IL-6 induced bFGF-dependent angiogenesis in basal cell carcinoma cell line via JAK/STAT3 and PI3K/Akt pathways.

5.2. Indirect Photobiomodulation. The most studied normal functions of cells are normal proliferation. LLL can upgrade the normal proliferation by promoting the activation of its partially activated rNSP.

In our experiments $[9,10]$, the LIL at $640 \mathrm{~nm}$ was found to induce the first-order phase transition from the first-order normal proliferation of $\mathrm{C} 2 \mathrm{C} 12$ myoblasts in a PISM to the second-order normal proliferation and then to the higherorder normal proliferation. For the first phase transition, the NSP and the first rNSP were found to be the nNSP and hNSP, respectively. The normal proliferation was maintained by the full activation of nNSP. LLL promoted hNSP activation. The synergistic action of nNSP and hNSP inhibited FOXO3a so that the normal proliferation was upgraded. FOXO3a inhibits breast cancer cell proliferation. The second phase transition from the second-order normal proliferation to the higherorder normal proliferation has been left to be solved.

For the iPBM on the normal proliferation of NIH3T3 fibroblasts in a PISM in 10\% FBS $[9,46]$, the rNSP is the PDGF-C pathway. Komine et al. [46] have studied LPBM on mRNA expression of PDGF-A, PDGF-B, PDGF-C, transforming growth factor-beta (TGF-beta), bFGF, PDGF-alpha receptor, and TGF-beta receptor and found that LPBM only increased PDGF-C mRNA expression but could not affect the mRNA expression of other genes.

For the iPBM on the normal proliferation of a human osteoblast-like cell line (Saos-2 cell line) in a PISM in 10\% FBS [47], the rNSP is one differentiation-mediated pathway. Bloise et al. [47] found that multiple doses of LIL increased extracellular matrix constituents such as alkaline phosphatase (ALP), decorin, fibronectin, and type-III collagen but did not affect extracellular matrix constituents such as osteocalcin, osteopontin, osteonectin, and type-I collagen. Moreover, multiple doses of LIL decreased the bone sialoprotein mRNA expression. On the other hand, the proliferation enhancement of titanium bioglass-coated scaffolds for Saos-2 human osteoblasts enhanced the deposition of extracellular matrix components such as ALP, decorin, fibronectin, osteocalcin, osteonectin, osteopontin, and type-I and -III collagens [48].

For the iPBM of the MIL at $532 \mathrm{~nm}$ on the normal proliferation of the human-derived glioblastoma cells (A-172) in a PISM in 10\% FBS [49] and the MIL at 660 or $780 \mathrm{~nm}$ on the normal proliferation of oral dysplastic cells in a PISM in $10 \%$ FBS [50], the rNSP is AKT-mediated pathway. The $\gamma$ secretase inhibitor (GSI) inhibits AKT activity and then A172 proliferation. Fukuzaki et al. [49] found that the MIL at $532 \mathrm{~nm}$ can promote GSI inhibited proliferation far from its PISH. For the MIL at 660 or $780 \mathrm{~nm}$ on oral dysplastic cells [50], the heat shock protein 90 level increased at $12 \mathrm{~h}$ but completely recovered at 48 and $72 \mathrm{~h}$.

For the iPBM on the normal proliferation of mouse bone marrow MSCs (D1 cells) in a PlSM [51], the rNSP is IGF1/bone morphogenetic protein-2 (BMP-2)/ALP mediated pathway. IGF-1 can upregulate BMP-2 [52] and then ALP [53] expression. ALP is essential for proliferation [54]. Wu et al. [51] have studied LPBM on the mRNA expression of IGF-1, ALP, BMP-2, osteocalcin, runt-related transcription factor 2, and receptor-activated nuclear factor kappa B ligand/osteoprotegerin on days 3 and 5 from the first LIL irradiation on and found that LPBM increased the mRNA expression of IGF-1 on days 3 and 5 and ALP on day 5 but could not affect the mRNA expression of other proteins. The iPBM promoted the proliferation from day $5 \mathrm{on}$. They directly found the mediation of IGF-1 and BMP-2 on LPBM although they cannot found LPBM effects on BIM-2 mRNA expression on days 3 and 5 .

The iPBM may be observed during the first irradiation [55] or just after the first irradiation [10] or some days after the first irradiation $[46,47,51,56]$. In other words, the iPBM may not be observed during the first irradiation [57] or just after the first irradiation [58]. The duration may be wavelengthdependent. Szymanska et al. [59] observed the iPBM of LIL at $635 \mathrm{~nm}$ but not $830 \mathrm{~nm}$ on the normal proliferation of vascular endothelial cells in 20\% FBS at day 4 .

Cellular factors may promote iPBM. Mvula et al. [60] have studied the LPBM on adipose-derived stem cells (ADSCs) in a PISM. Either epidermal growth factor (EGF) or one time LIL irradiation alone cannot affect ADSC proliferation, but their integration upgraded the normal proliferation at $48 \mathrm{~h}$ after the irradiation. For the $\mathrm{PBBM}$ on ADSCs in a PISM [61], the rNSP is betal-integrin mediated pathway. There is indeed an integration of IGF-1 and betal-integrin [62].

One time irradiation may be self-limited. Bloise et al. [47] observed the iPBM of multiple doses of LIL on the normal proliferation of the Saos- 2 cell line in a PISM, but they found that the effects of single dose were self-limited.

\section{Discussions}

As it has been pointed out in this paper, which function of a cell may be perfectly performed depends on the microenvironment in which cells live. The dependence may be thermodynamic. A PSM allowed proliferation may be a direct proliferation through its NSP or a differentiationmediated proliferation through its rNSP for $\mathrm{APBM}$ or their synergistic integration for iPBM. For an iPBM in a PISM, the differentiation was first promoted [51], but the normal 
proliferation was finally upgraded. A DSM allowed differentiation may also be a direct differentiation through its NSP or a proliferation-mediated differentiation through its rNSP. MSCs were collected from adult human bone marrow, isolated, and precultured in complete medium and cultured in osteogenic medium in three-dimensional collagen scaffolds and simultaneously irradiated with LIL. Leonida et al. [63] found that the LIL promoted both proliferation and differentiation at day 7 but only promoted differentiation at day 14. Obviously, the proliferation at day 7 was differentiationmediated, but the differentiation at day 14 was proliferationmediated.

One signal transduction pathway may directly mediate normal differentiation in a DSM as an NSP in a DPBM and may also indirectly mediate enhanced normal proliferation through differentiation in a PISM as an rNSP of the NSP of the normal proliferation in an iPBM. It was found that the promotion of LIL at $685 \mathrm{~nm}$ on both the osteoblast differentiation of human MSCs in osteogenic medium which included 10\% FBS and $100 \mathrm{nM}$ DEX in its $\mathrm{APBM}$ [39] and proliferation of human gingival fibroblasts in a PISM in 10\% FBS in its iPBM [64] was mediated by bFGF/IGF-1/IGFBP3 mediated pathway. Pons and Torres-Aleman [65] found that bFGF strongly modulates IGF-1, its receptors, and its binding proteins in the two major cell types of the hypothalamus.

DEX can inhibit proliferation. It might inhibit one NSP, but one of its rNSPs can be fully activated to maintain normal proliferation. Wu et al. [66] found that LPBM can promote DEX inhibited proliferation of human periodontal ligament cells through differentiation-mediated pathway which included BMP-2 and ALP. The pathway might include IGF-1 according to Wu et al. [51] as it has been discussed in Section 5.2.

\section{Conclusions}

One normal function may have $N$ possible NSPs which are redundant with one another. The first-order normal function is maintained by the full activation of each NSP in its NSM, but its enhancement, for example, the $n$ th-order normal function $(n=2,3, \ldots, N)$, is maintained by the synergistic full activation of the NSP and its $n-1$ rNSPs in its NSM. A LLL may promote the activation of partially activated NSP or/and its rNSP so that a dPBM may induce the second-order functional phase transition from a dysfunctional function to the first-order normal function in a function-specific microenvironment, and an iPBM may also induce the firstorder functional phase transition from the $(n-1)$ th-order normal function to the $n$ th-order normal function in its NSM.

\section{Conflict of Interests}

The authors declare that there is no conflict of interests regarding the publication of this paper.

\section{Authors' Contribution}

T. C.-Y. Liu and D.-F. Wu contributed equally to this work.

\section{Acknowledgments}

This work was supported by National Science Foundation of China (60878061, 10974061, and 11374107), Doctoral Fund of Ministry of Education of China (20124407110013), and Guangdong Scientific Project (2012B031600004).

\section{References}

[1] E. S. Valchinov and N. E. Pallikarakis, "Design and testing of low intensity laser biostimulator," BioMedical Engineering Online, vol. 4, article 5, 2005.

[2] H. Chung, T. Dai, S. K. Sharma, Y. Y. Huang, J. D. Carroll, and M. R. Hamblin, "The nuts and bolts of low-level laser (light) therapy," Annals of Biomedical Engineering, vol. 40, no. 2, pp. 516-533, 2012.

[3] C. Y. Liu and P. Zhu, Intranasal Low Intensity Laser Therapy, People's Military Medical Press, Beijing, China, 2009.

[4] T. C. Y. Liu, R. Liu, L. Zhu, J. Q. Yuan, M. Wu, and S. H. Liu, "Homeostatic photobiomodulation," Front Optoelectron China, vol. 2, no. 1, pp. 1-8, 2009.

[5] T. C. Y. Liu, D. F. Wu, Z. Q. Gu, and M. Wu, "Applications of intranasal low intensity laser therapy in sports medicine," Journal of Innovative Optical Health Sciences, vol. 3, no. 1, pp. $1-16,2010$.

[6] L. Baratto, L. Calzà, R. Capra et al., "Ultra-low-level laser therapy," Lasers in Medical Science, vol. 26, no. 1, pp. 103-112, 2011.

[7] J. L. Hartman IV, B. Garvik, and L. Hartwell, "Principles for the buffering of genetic variation," Science, vol. 291, no. 5506, pp. 1001-1004, 2001.

[8] T. C. Y. Liu, Y. Y. Liu, E. X. Wei, and F. H. Li, "Photobiomodulation on stress," International Journal of Photoenergy, vol. 2012, Article ID 628649, 11 pages, 2012.

[9] T. C. Y. Liu, L. Zhu, and X. B. Yang, "Photobiomodulationmediated pathway diagnostics," Journal of Innovation in Optical Health Science, vol. 6, no. 1, Article ID 1330001, 11 pages, 2013.

[10] T. C. Y. Liu, F. H. Li, E. X. Wei, and L. Y. Deng, "Redundant pathway mediated photobiomodulation enhancement on a myoblast proliferation in its proliferation-specific homeostasis," Lasers in Surgery and Medicine, vol. 45, supplement 25, p. 53, 2013.

[11] D. F. Wu, The cross talk between proliferation and clock gene expression of serum-shocked myoblasts and its mechanism [Ph.D. thesis], South China Normal University, 2014.

[12] L. Y. Deng, Self-limited recovery of high glucose induced stress of mouse mesenchymal stem cells and its photobiomodulation [M.S. thesis], South China Normal University, 2013.

[13] R. Straussman, T. Morikawa, K. Shee et al., "Tumour microenvironment elicits innate resistance to RAF inhibitors through HGF secretion," Nature, vol. 487, no. 7408, pp. 500-504, 2012.

[14] B. Haibe-Kains, N. El-Hachem, N. J. Birkbak et al., "Inconsistency in large pharmacogenomic studies," Nature, vol. 504, no. 7480, pp. 389-393, 2013.

[15] M. Wu and T. C. Y. Liu, "Single-cell analysis of protein kinase C activation during anti-apoptosis and apoptosis induced by laser irradiation," Photomedicine and Laser Surgery, vol. 25, no. 2, pp. 129-130, 2007.

[16] M. A. Pogrel, J. W. Chen, and K. Zhang, "Effects of lowenergy gallium-aluminum-arsenide laser irradiation on cultured fibroblasts and keratinocytes," Lasers in Surgery and Medicine, vol. 20, no. 4, pp. 426-432, 1997. 
[17] H. Miyata, T. Genma, M. Ohshima et al., "Mitogen-activated protein kinase/extracellular signal-regulated protein kinase activation of cultured human dental pulp cells by lowpower gallium-aluminium-arsenic laser irradiation," International Endodontic Journal, vol. 39, no. 3, pp. 238-244, 2006.

[18] F. D. P. Eduardo, D. F. Bueno, P. M. de Freitas et al., "Stem cell proliferation under low intensity laser irradiation: A Preliminary Study," Lasers in Surgery and Medicine, vol. 40, no. 6, pp. 433-438, 2008.

[19] R. Duan, L. Zhu, T. C. Y. Liu et al., "Light emitting diode irradiation protect against the amyloid beta 25-35 induced apoptosis of PC12 cell in vitro," Lasers in Surgery and Medicine, vol. 33, no. 3, pp. 199-203, 2003.

[20] L. Zhu, X. Y. Li, T. C. Y. Liu, and S. H. Liu, "Protective effects of red light on differentiated PC12 cells against hydrogen peroxide induced oxidative stress," Lasers in Surgery and Medicine, vol. 41, supplement 21, p. 60, 2009.

[21] P. C. Davies, L. Demetrius, and J. A. Tuszynski, "Cancer as a dynamical phase transition," Theoretical Biology and Medical Modelling, vol. 8, article 30, 2011.

[22] E. D. Levy, C. R. Landry, and S. W. Michnick, "Cell signaling: signaling through cooperation," Science, vol. 328, no. 5981, pp. 983-984, 2010.

[23] D. Chrysis, A. Chagin, and L. Sävendahl, "Insulin-like growth factor-1 restores dexamethasone-induced heart growth arrest in rats: the role of the ubiquitin pathway," Hormones, vol. 10, no. 1, pp. 46-56, 2011.

[24] F. Giorgino and R. J. Smith, "Dexamethasone enhances insulinlike growth factor-I effects on skeletal muscle cell proliferation. Role of specific intracellular signaling pathways," Journal of Clinical Investigation, vol. 96, no. 3, pp. 1473-1483, 1995.

[25] Y. Uchijima, A. Takenaka, S. Takahashi, and T. Noguchi, "Dexamethasone stabilizes IGFBP-1 mRNA in primary cultures of rat hepatocytes," Endocrine Journal, vol. 46, no. 3, pp. 471-476, 1999.

[26] M. A. Nowak, M. C. Boerlijst, J. Cooke, and J. M. Smith, "Evolution of genetic redundancy," Nature, vol. 388, no. 6638, pp. 167-171, 1997.

[27] D. A. Linseman, R. A. Phelps, R. J. Bouchard et al., "Insulin-like growth factor-I blocks Bcl-2 interacting mediator of cell death (Bim) induction and intrinsic death signaling in cerebellar granule neurons," Journal of Neuroscience, vol. 22, no. 21, pp. 9287-9297, 2002.

[28] E. de Bruyne, T. J. Bos, F. Schuit et al., "IGF-1 suppresses Bim expression in multiple myeloma via epigenetic and posttranslational mechanisms," Blood, vol. 115, no. 12, pp. 2430-2440, 2010.

[29] N. Lane, “Cell biology: power games," Nature, vol. 443, no. 7114, pp. 901-903, 2006.

[30] M. Hüttemann, I. Lee, L. I. Grossman, J. W. Doan, and T. H. Sanderson, "Phosphorylation of mammalian cytochrome $c$ and cytochrome $c$ oxidase in the regulation of cell destiny: respiration, apoptosis, and human disease," Advances in Experimental Medicine and Biology, vol. 748, pp. 237-264, 2012.

[31] T. I. Karu and N. I. Afanas'eva, "Cytochrome $c$ oxidase as the primary photoacceptor upon laser exposure of cultured cells to visible and near IR-range light," Doklady Akademii Nauk, vol. 342, no. 5, pp. 693-695, 1995.

[32] T. Karu, The Science of Low-Power Laser Therapy, Gordon and Breach Science Publishers, Amsterdam, The Netherlands, 1998.

[33] S. Song, F. Zhou, and W. R. Chen, "Low-level laser therapy regulates microglial function through $\mathrm{Src}$-mediated signaling pathways: implications for neurodegenerative diseases," Journal of Neuroinflammation, vol. 9, article 219, 2012.

[34] Y. Y. Huang, K. Nagata, C. E. Tedford, and M. R. Hamblin, "Lowlevel laser therapy $(810 \mathrm{~nm})$ protects primary cortical neurons against excitotoxicity in vitro," Journal of Biophotonics, 2014.

[35] L. A. Montoro, A. P. Turrioni, F. G. Basso, C. A. de Souza Costa, and J. Hebling, "Infrared LED irradiation photobiomodulation of oxidative stress in human dental pulp cells," International Endodontic Journal, 2014.

[36] S. Wu, F. Zhou, Y. Wei, W. R. Chen, Q. Chen, and D. Xing, "Cancer phototherapy via selective photoinactivation of respiratory chain oxidase to trigger a fatal superoxide anion burst," Antioxidants \& Redox Signaling, 2014.

[37] K. Horvát-Karajz, Z. Balogh, V. Kovács, A. H. Drrernat, L. Sréter, and F. Uher, "In vitro effect of carboplatin, cytarabine, paclitaxel, vincristine, and low-power laser irradiation on murine mesenchymal stem cells," Lasers in Surgery and Medicine, vol. 41, no. 6, pp. 463-469, 2009.

[38] Y. Y. Xu, T. C. Y. Liu, and L. Cheng, "Photobiomodulation process," International Journal of Photoenergy, vol. 2012, Article ID 374861, 7 pages, 2012.

[39] I. Saygun, N. Nizam, A. U. Ural, M. A. Serdar, F. Avcu, and T. F. Tözüm, "Low-level laser irradiation affects the release of basic fibroblast growth factor (bFGF), insulin-like growth factorI (IGF-I), and receptor of IGF-I (IGFBP3) from osteoblasts," Photomedicine and Laser Surgery, vol. 30, no. 3, pp. 149-154, 2012.

[40] C. Meng, Z. He, and D. Xing, "Low-level laser therapy rescues dendrite atrophy via upregulating BDNF expression: implications for Alzheimer's disease," Journal of Neuroscience, vol. 33, no. 33, pp. 13505-13517, 2013.

[41] S. T. Nam, D. H. Kim, M. B. Lee et al., "Insect peptide CopA3induced protein degradation of $\mathrm{p} 27 \mathrm{Kip} 1$ stimulates proliferation and protects neuronal cells from apoptosis," Biochemical and Biophysical Research Communications, vol. 437, no. 1, pp. 35-40, 2013.

[42] B. Y. Chen, X. Wang, Z. Y. Wang, Y. Z. Wang, L. W. Chen, and Z. J. Luo, "Brain-derived neurotrophic factor stimulates proliferation and differentiation of neural stem cells, possibly by triggering the Wnt/ $\beta$-catenin signaling pathway," Journal of Neuroscience Research, vol. 91, no. 1, pp. 30-41, 2013.

[43] Y. Y. Huang, K. Nagata, C. E. Tedford, T. McCarthy, and M. R. Hamblin, "Low-level laser therapy (LLLT) reduces oxidative stress in primary cortical neurons in vitro," Journal of Biophotonics, vol. 6, no. 10, pp. 829-838, 2013.

[44] M. Esmaeelinejad and M. Bayat, "Effect of low-level laser therapy on the release of interleukin- 6 and basic fibroblast growth factor from cultured human skin fibroblasts in normal and high glucose mediums," Journal of Cosmetic and Laser Therapy, vol. 15, no. 6, pp. 310-317, 2013.

[45] S. H. Jee, C. Y. Chu, H. C. Chiu et al., "Interleukin-6 induced basic fibroblast growth factor-dependent angiogenesis in basal cell carcinoma cell line via JAK/STAT3 and PI3-kinase/Akt pathways," Journal of Investigative Dermatology, vol. 123, no. 6, pp. 1169-1175, 2004.

[46] N. Komine, K. Ikeda, K. Tada, N. Hashimoto, N. Sugimoto, and K. Tomita, "Activation of the extracellular signal-regulated kinase signal pathway by light emitting diode irradiation," Lasers in Medical Science, vol. 25, no. 4, pp. 531-537, 2010.

[47] N. Bloise, G. Ceccarelli, P. Minzioni et al., "Investigation of lowlevel laser therapy potentiality on proliferation and differentiation of human osteoblast-like cells in the absence/presence of 
osteogenic factors," Journal of Biomedical Optics, vol. 18, no. 12, Article ID 128006, 2013.

[48] E. Saino, V. Maliardi, E. Quartarone et al., "In vitro enhancement of SAOS-2 cell calcified matrix deposition onto radio frequency magnetron sputtered bioglass-coated titanium scaffolds," Tissue Engineering A, vol. 16, no. 3, pp. 995-1008, 2010.

[49] Y. Fukuzaki, H. Sugawara, B. Yamanoha, and S. Kogure, “532 nm low-power laser irradiation recovers $\gamma$-secretase inhibitormediated cell growth suppression and promotes cell proliferation via Akt signaling," PLoS ONE, vol. 8, no. 8, Article ID e70737, 2013.

[50] F. F. Sperandio, F. S. Giudice, L. Corrêa, D. S. Pinto Jr., M. R. Hamblin, and S. C. de Sousa, "Low-level laser therapy can produce increased aggressiveness of dysplastic and oral cancer cell lines by modulation of Akt/mTOR signaling pathway," Journal of Biophotonics, vol. 6, no. 10, pp. 839-847, 2013.

[51] J. Y. Wu, Y. H. Wang, G. J. Wang et al., "Low-power GaAlAs laser irradiation promotes the proliferation and osteogenic differentiation of stem cells via IGF1 and BMP2," PLoS ONE, vol. 7, no. 9, Article ID e44027, 2012.

[52] G. J. Xu, S. Cai, and J. B. Wu, "Effect of insulin-like growth factor-1 on bone morphogenetic protein-2 expression in hepatic carcinoma SMMC7721 cells through the p38 MAPK signaling pathway," Asian Pacific Journal of Cancer Prevention, vol. 13, no. 4, pp. 1183-1186, 2012.

[53] Y. J. Kim, M. H. Lee, J. M. Wozney, J. Y. Cho, and H. M. Ryoo, "Bone morphogenetic protein-2-induced alkaline phosphatase expression is stimulated by Dlx5 and repressed by Msx2," Journal of Biological Chemistry, vol. 279, no. 49, pp. 5077350780, 2004.

[54] V. Kermer, M. Ritter, B. Albuquerque, C. Leib, M. Stanke, and H. Zimmermann, "Knockdown of tissue nonspecific alkaline phosphatase impairs neural stem cell proliferation and differentiation," Neuroscience Letters, vol. 485, no. 3, pp. 208-211, 2010.

[55] T. Kantermann, S. Forstner, M. Halle, L. Schlangen, T. Roenneberg, and A. Schmidt-Trucksäss, "The stimulating effect of bright light on physical performance depends on internal time," PLoS ONE, vol. 7, no. 7, Article ID e40655, 2012.

[56] W. P. Hu, J. J. Wang, C. L. Yu, C. C. E. Lan, G. S. Chen, and H. S. $\mathrm{Yu}$, "Helium-neon laser irradiation stimulates cell proliferation through photostimulatory effects in mitochondria," Journal of Investigative Dermatology, vol. 127, no. 8, pp. 2048-2057, 2007.

[57] P. M. O'Brien and P. J. O'Connor, "Effect of bright light on cycling performance," Medicine and Science in Sports and Exercise, vol. 32, no. 2, pp. 439-447, 2000.

[58] S. Incerti Parenti, S. Panseri, A. Gracco, M. Sandri, A. Tampieri, and G. A. Bonetti, "Effect of low-level laser irradiation on osteoblast-like cells cultured on porous hydroxyapatite scaffolds," Annali dell'Istituto Superiore di Sanità, vol. 49, no. 3, pp. 255-260, 2013.

[59] J. Szymanska, K. Goralczyk, J. J. Klawe et al., "Phototherapy with low-level laser influences the proliferation of endothelial cells and vascular endothelial growth factor and transforming growth factor-beta secretion," Journal of Physiology and Pharmacology, vol. 64, no. 3, pp. 387-391, 2013.

[60] B. Mvula, T. J. Moore, and H. Abrahamse, "Effect of low-level laser irradiation and epidermal growth factor on adult human adipose-derived stem cells," Lasers in Medical Science, vol. 25, no. 1, pp. 33-39, 2010.

[61] B. Mvula, T. Mathope, T. Moore, and H. Abrahamse, "The effect of low level laser irradiation on adult human adipose derived stem cells," Lasers in Medical Science, vol. 23, no. 3, pp. 277-282, 2008.

[62] Y. Suzuki, M. Yanagisawa, H. Yagi, Y. Nakatani, and R. K. Yu, "Involvement of $\beta$-integrin up-regulation in basic fibroblast growth factor- and epidermal growth factor-induced proliferation of mouse neuroepithelial cells," Journal of Biological Chemistry, vol. 285, no. 24, pp. 18443-18451, 2010.

[63] A. Leonida, A. Paiusco, G. Rossi, F. Carini, M. Baldoni, and G. Caccianiga, "Effects of low-level laser irradiation on proliferation and osteoblastic differentiation of human mesenchymal stem cells seeded on a three-dimensional biomatrix: in vitro pilot study," Lasers in Medical Science, vol. 28, no. 1, pp. 125-132, 2013.

[64] I. Saygun, S. Karacay, M. Serdar, A. U. Ural, M. Sencimen, and B. Kurtis, "Effects of laser irradiation on the release of basic fibroblast growth factor (bFGF), insulin like growth factor-1 (IGF-1), and receptor of IGF-1 (IGFBP3) from gingival fibroblasts," Lasers in Medical Science, vol. 23, no. 2, pp. 211-215, 2008.

[65] S. Pons and I. Torres-Aleman, "Basic fibroblast growth factor modulates insulin-like growth factor-I, its receptor, and its binding proteins in hypothalamic cell cultures," Endocrinology, vol. 131, no. 5, pp. 2271-2278, 1992.

[66] J. Y. Wu, C. H. Chen, L. Y. Yeh, M. L. Yeh, C. C. Ting, and Y. H. Wang, "Low-power laser irradiation promotes the proliferation and osteogenic differentiation of human periodontal ligament cells via cyclic adenosine monophosphate," International Journal of Oral Science, vol. 5, no. 2, pp. 85-91, 2013. 

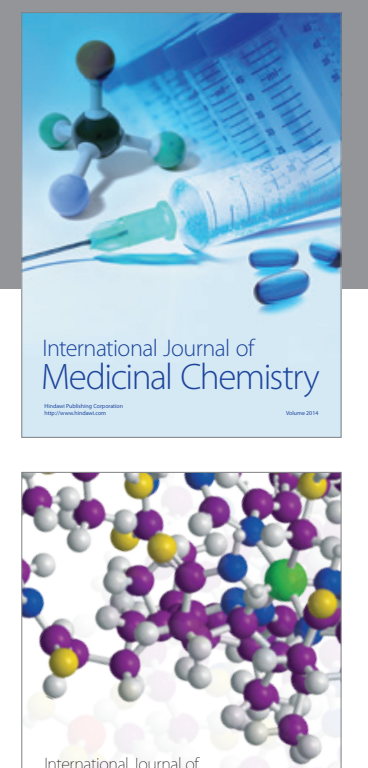

\section{Carbohydrate} Chemistry

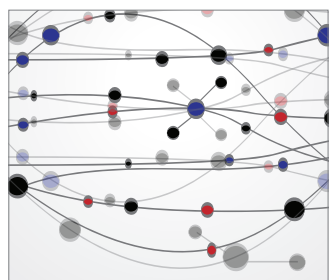

The Scientific World Journal
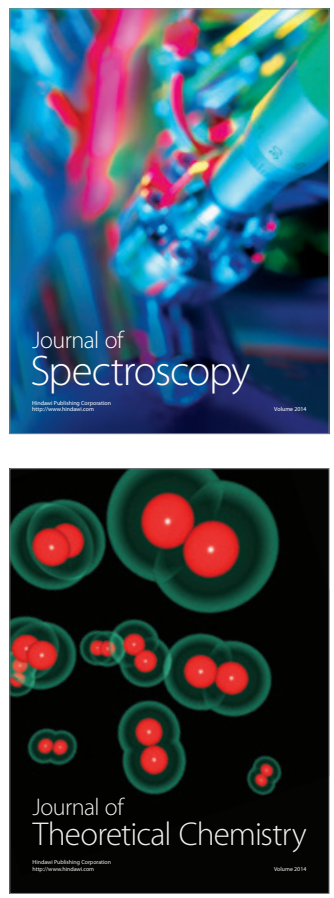
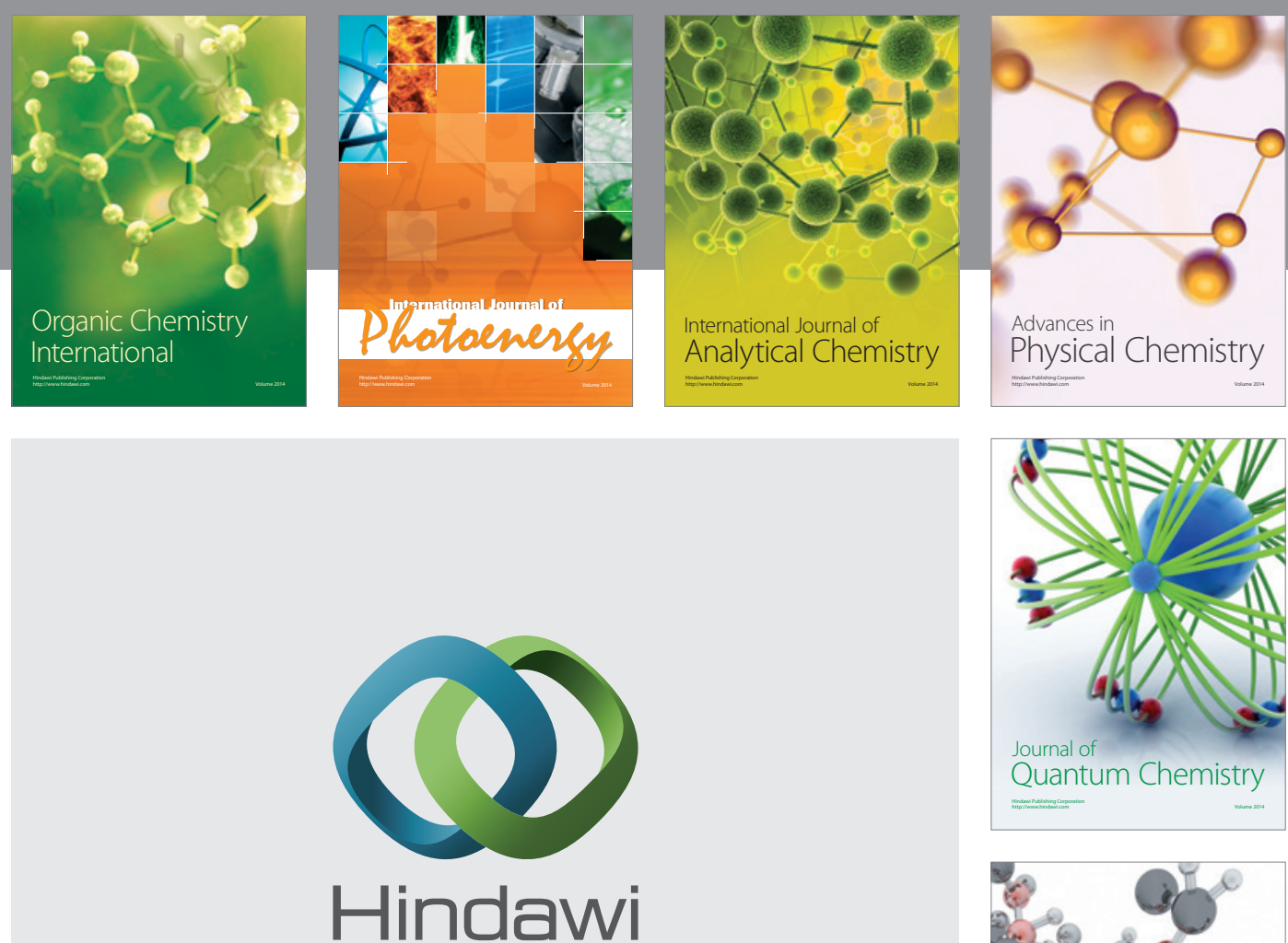

Submit your manuscripts at

http://www.hindawi.com

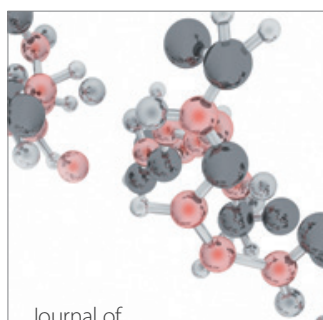

Analytical Methods

in Chemistry

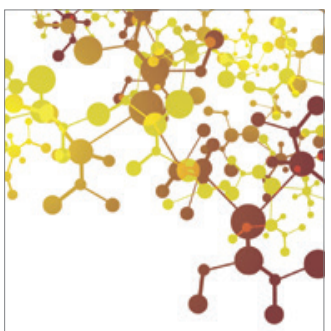

Journal of

Applied Chemistry

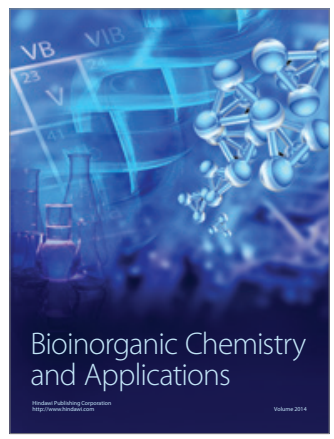

Inorganic Chemistry
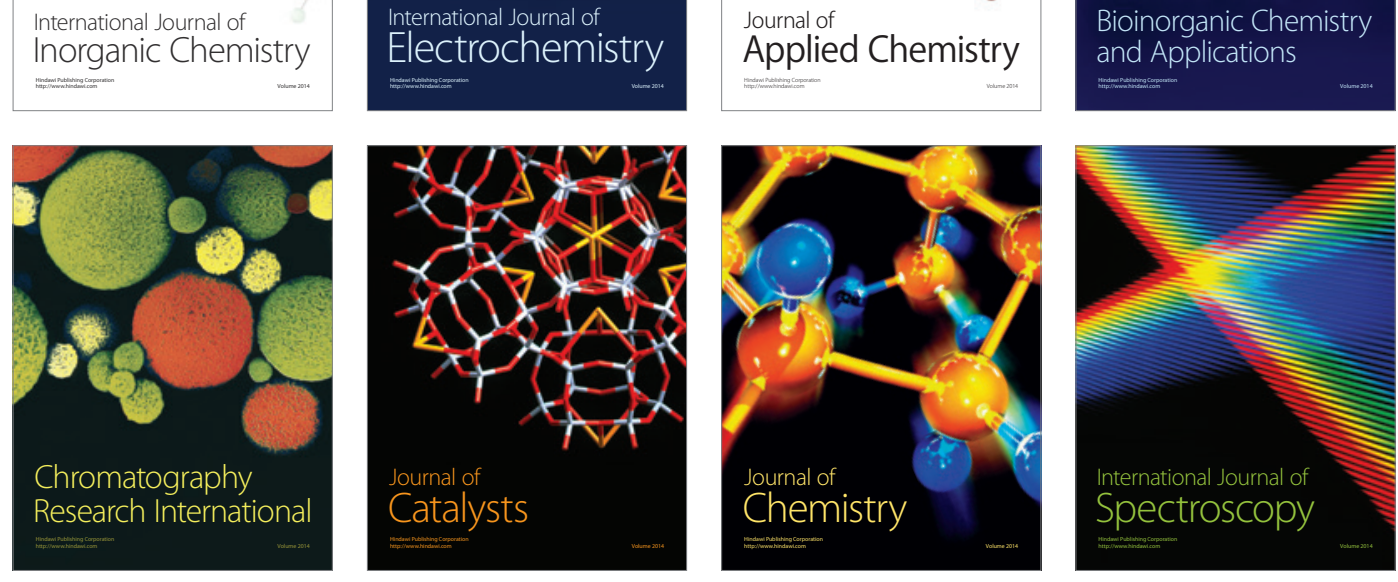